\title{
West Nile virus
}

Background and epidemiology: West Nile virus (WNV) is a single-stranded RNA virus of the Flaviviridae family. It is maintained in nature by a cycle involving mosquitoes and birds that occasionally spills over to humans. Outbreaks in humans were first reported in 1937 in Uganda and subsequently recorded in Egypt, Israel, South Africa, Europe and Asia. The virus arrived in North America in 1999, probably through an infected migratory or imported bird or an infected mosquito.

The first cases of infection in humans in Canada were documented in 2002, and by 2003 WNV was present in 7 provinces, with more than 1300 cases confirmed. ${ }^{1}$ By 2004 local governments in many of the hot spots of WNV activity in Canada were applying larvicides to control the population of mosquito vectors. This may explain the dramatic reduction in human cases observed in 2004: only 26 cases in total were reported in Quebec, Ontario, Manitoba, Saskatchewan and Albert, of which 1 was apparently travel-related.

At the time of writing (Aug. $3,2005)$ there have been 24 confirmed cases of infection in birds and 1 unconfirmed in a human this year (www.phacaspc.gc.ca/wnv-vwn/). However, because WNV activity typically increases in late summer and early fall and is influenced by many factors, including weather conditions and the number of infected birds and mosquitoes in a region, it is too early to draw conclusions about the level of $\mathrm{WNV}$ activity this year.

Clinical management: The incubation period is 3-14 days. Many people remain asymptomatic. In about $20 \%$ of cases there is a flu-like illness of sudden onset with symptoms that can include malaise, headache, anorexia, nausea, vomiting, eye pain, myalgia and lymphadenopathy. A few patients experience a rash, which can be an erythematous macular, papular or morbilliform eruption involving the neck, trunk, arms or legs. ${ }^{2}$ Symptoms typically last 3-6 days.

In about 1 in 150 patients the infection will develop into meningitis, encephalitis or both. This is more common with advancing age and immune system suppression. Other, rarer presentations include ataxia, cranial nerve abnormalities, optic neuritis, myelitis and seizures. ${ }^{2}$ Case-fatality rates range from $4 \%$ to $14 \%$.

WNV infection should be strongly considered in adults over 50 years with unexplained encephalitis or meningitis in summer or early fall. Patient travel history and an awareness of local levels of WNV activity are important. WNV has also spread through blood and organ donation, needle-stick injury and exposure to infected laboratory specimens. Pregnant women may pass the virus on to their fetuses, and the virus can be passed through breast milk. ${ }^{2}$

Diagnosis: The most commonly used test is detection of $\operatorname{IgM}$ to $\mathrm{WNV}$ in serum or cerebrospinal fluid using enzyme-linked immunoabsorbent assay (ELISA) testing.

Test cross-reactivity may cause false-positive results after other flaviviral infections or vaccination such as yellow fever or Japanese encephalitis. Also, serum IgM persists for up to 12 months after infection, and physicians must determine whether positive results are due to a recent infection or unrelated to the current presentation. Repeat ELISA testing of paired acute and convalescent sera 7-14 days apart will show a 4-fold rise in acute infection. The plaque reduction neutralization test, specific for arthro- pod-borne flaviviruses, can also be used to help distinguish falsepositive results. ${ }^{2}$ Testing is available through provincial health departments.

Treatment consists of supportive care. Trials of ribavirin and interferon $\alpha-2 b$ have not been completed. ${ }^{2}$

Prevention: There is no vaccine against WNV infection. Prevention depends on using mosquito repellent, avoiding peak biting times and wearing protective clothing such as longsleeved shirts, trousers and socks. People should avoid handling dead birds and animals that may be infected.

Insect repellents should be used with caution. Children under 12 years should not be exposed to DEET concentrations of more than $10 \%$, and DEET should not be used on infants less than 6 months. Permethrin should not be applied directly to the skin. ${ }^{3}$ Spraying clothing with DEET or permethrin-containing repellent offers extra protection.

Eliminating mosquito breeding grounds is also important, and standing water should be removed from outdoor containers. Local councils may also undertake mosquito control using larvicides and adulticides.

$\mathrm{WNV}$ is a notifiable disease, and rapid reporting of possible cases is essential to guide public health control efforts.

Sally Murray

Erica Weir

CMA7

\section{References}

1. Nosal B, Pellizzari R. West Nile virus. CMA7 2003;168(11):1443.

2. Centre for Disease Control. West Nile virus infection: information for clinicians. Available: www.cdc.gov/ncidod /dvbid/westnile/clinicians/treatment.htm (accessed 2005 Jul 18).

3. Public Health Agency of Canada. Safety tips on using personal insect repellents. Available: www.hc-sc.gc.ca /hpb/lcdc/wn-no/repellents-insectifuge _e.html (accessed 2005 Jul 18). 